\title{
Discriminability in length of lines in the Müller-Lyer figure
}

\author{
MIAO-FEN WANG, R. JOHN IRWIN, and MICHAEL J. HAUTUS \\ University of Auckland, Auckland, New Zealand
}

\begin{abstract}
Receiver-operating characteristics for the discriminability in the length of the lines of the Müller-Lyer figure were obtained by the rating method of detection theory. Six observers judged the shaft length of the lines of the figure with reference to the same standard line. Maximum-likelihood estimates of the index of discriminability, $d^{\prime}$, were a linear function of the difference in the length of the lines, but the functions did not pass through the origin because of a constant error of judgment. Because discriminability was determined by ROC analysis, the constant error could not be attributed solely to changes in criterion placement; instead, it showed that the Müller-Lyer figure induced a change in the discriminability of the lines.
\end{abstract}

Despite its apparent relevance, detection theory has seldom been used to elucidate the processes underlying visual illusions. Here we attempt to show how detection theory can help clarify some of the factors that underlie the Müller-Lyer illusion. There are many variants of this famous illusion (see Day \& Knuth, 1981, for some history), but the prototypical example consists of two lines of equal length, presented side-by-side, in which one of the lines has, in addition, either arrowheads $(<>)$ or tail fins $(><)$ on each end. Arrowheads make the line appear shorter than a physically equal reference line, and fins make it appear longer.

A vast number of investigations have attempted to uncover the causes of this illusion. We find Coren and Girgus's (1978) classification of theories into two typesstructural theories and strategy theories - to be of value. Briefly, structural theories attribute the illusion to optical or neural processes. For example, Ginsburg (1986) explained the illusion in terms of the limited capacity of the visual system to transmit the spatial frequencies that make up the Müller-Lyer figure. Strategy theories ascribe the illusion to cognitive processes-for example, "to biases introduced by judgmental and attentional processes" (Coren \& Girgus, p. 73). Pressey and his colleagues (e.g., Pressey \& Pressey, 1992) have explained the illusion as stemming from the deployment of an attentive field-a deployment that may be, at least partly, at the disposal of the observer.

Nevin (1991) made an insightful observation by identifying these two kinds of theories with the two independent processes measured by detection theory: discriminability and response bias. If this identification is valid, structural causes ought to show up as affecting the dis-

We thank Tony Nevin and two other referees for their thoughtful and challenging reviews of previous versions of our manuscript. Correspondence concerning this article should be addressed to R. J. Irwin, Department of Psychology, The University of Auckland, Private Bag 92019. Auckland, New Zealand (e-mail: rji@auckland.ac.nz). criminability of aspects of the stimulus, whereas cognitive causes ought to be manifested in changes in an observer's criterion for reporting the illusory effect. Therefore, by analyzing the Müller-Lyer illusion with the concepts of detection theory, Nevin reasoned that it might be possible to parcel out the contribution to the illusion of those cognitive processes that are reflected in response biases and the contribution of those structural processes that are reflected in discrimination. Such an analysis, coupled with the psychoanatomical dissection suggested by Coren and Girgus (1978), might identify more precisely the nature of the structural or cognitive processes that contribute to the illusion. Therefore, a detection-theoretic analysis of illusory experiences ought to offer a new insight, even if not a definitive one, into the origins of illusions, because of its ability to separate changes in discriminability from changes in criterion placement.

There are, however, some issues that complicate the application of detection theory to the understanding of visual illusions. These issues can be highlighted through a description of two previous attempts to undertake a detection-theoretic analysis of the Müller-Lyer illusion.

\section{Detection-Theoretic Analyses of the Müller-Lyer Illusion}

Lown (1988) may have been the first to apply detection theory to the measurement of the Müller-Lyer illusion. In his investigation, each trial contained both a standard and a comparison line. The standard line had arrowheads at each end, and the comparison line had fins. The standard line was always $100 \mathrm{~mm}$ long. On "noise" trials, the comparison line was $75 \mathrm{~mm}$ long (it was then judged to be equal to the standard line), and on "signal" trials, it was $80,90,100$, or $110 \mathrm{~mm}$ long. On each trial, "a subject was required to say whether the line with fins out (comparison) was equal to or greater than the standard" (Lown, p. 101). In order to compute the detection-theory index of discriminability, $d^{\prime}$, the frequency with which observers judged the comparison line to be longer on noise trials 
was compared with the frequency with which they judged it to be longer on signal trials. The index so obtained measured the extent to which lines of $80,90,100$, or $110 \mathrm{~mm}$ were discriminable from a line of $75 \mathrm{~mm}$ when all the lines had tail fins. The stimulus-response matrix for Lown's experiment is depicted in Table 1, where we have labeled the noise as S1 and the signal as S2. The standard line with arrowheads served as a reference line for judging the length of the comparison line. Inspection of the stimulus arrangement in Table 1, however, shows that the relation between $p$ ("longer" $\mid \mathrm{S} 1$ ) and $p$ ("longer" $\mid \mathrm{S} 2$ ) does not quantify the magnitude of the Müller-Lyer illusion. Rather, it quantifies the extent to which one line that is terminated with fins is discriminable from another line that is terminated with fins. Despite its objective, therefore, Lown's study does not provide a detection-theoretic analysis of the illusion. (A similar study that derived nonparametric indices of discrimination has been reported by Brosvic et al., 1994.)

Nevin (1991) conducted an experiment whose design was a substantial improvement over Lown's (1988). As in Lown's design, every trial contained both a standard and a comparison line, displayed contiguously side-byside. There were two kinds of trials. In one kind, the two lines had short vertical bars at each end. Such a figure does not give rise to an illusory effect. In the other kind of trial, the standard line had arrowheads at each end, and the comparison line had fins. This second kind of trial, therefore, presented one of the normal versions of the Müller-Lyer illusion. After every trial, the observers judged whether the comparison line was longer or shorter than the standard. On both kinds of trials, illusory and nonillusory, the comparison line could take on one of eight lengths, whereas the standard line was always the same length. The stimulus-response matrix for Nevin's experiment is shown in Table 2.

Nevin (1991) did not attempt to compute a detectiontheoretic index of discriminability. Instead, Nevin derived separate psychometric functions for the stimuli presented on illusory trials (S2) and for those presented on nonillusory trials (S1). These functions showed how the ratio of "longer" to "shorter" judgments for each kind of trial depended on the difference in length between the comparison and the standard lines, which also was expressed as a ratio. He found that the slopes of the obtained functions were the same for both illusory and nonillusory configurations of the lines. From this, Nevin concluded that the Müller-Lyer configuration does not cause a change in the discriminability of line length. In our

Table 1

The Stimulus-Response Matrix for Lown's (1988) Experiment and the Possible Outcomes of a Trial

\begin{tabular}{|c|c|c|c|c|}
\hline \multirow[b]{2}{*}{ Stimulus } & \multicolumn{2}{|c|}{ Stimulus } & \multicolumn{2}{|c|}{ Response } \\
\hline & Standard & Comparison & "Co longer" & "Co equal" \\
\hline S1 & $\longleftrightarrow$ & 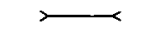 & $p$ ("longer"| $\mid \mathrm{S} 1)$ & $p$ ("equal"|SI) \\
\hline $\mathrm{S} 2$ & $\longleftrightarrow$ & $\Longleftarrow$ & $p$ (“longer" $\mid \mathrm{S} 2)$ & $p$ (“equal"| $\mid \mathrm{S} 2)$ \\
\hline
\end{tabular}

Table 2

The Stimulus-Response Matrix for Nevin's (1991) Experiment

\begin{tabular}{cccccc} 
& \multicolumn{2}{c}{ Stimulus } & & \multicolumn{2}{c}{ Response } \\
\cline { 2 - 3 } & Standard & Comparison & "Co longer" & "Co shorter" \\
\hline S1 & $\vdash$ & $\longmapsto$ & $p$ ("longer"|S1) & $p$ ("shorter"|S1) \\
S2 & $\longrightarrow$ & $\longmapsto$ & ("longer"|S2) & $p$ ("shorter"|S2)
\end{tabular}

Note-Co, comparison.

view, however, the slope of Nevin's functions indicates how discriminable in length one line is from another when both lines have identical terminations-that is, when no illusory effect is observable. For this reason, we suggest that in order to assess the illusory effect, Nevin's psychometric functions could have been constructed from the ratios of the responses to $S 1$ and $S 2$.

Nevin (1991) found that the horizontal location of the functions for the illusory configuration was displaced relative to that for the nonillusory configuration. He characterized this displacement in position as stemming from a change in response bias. This is an important conclusion because, taken in conjunction with the finding that there was no change in the discriminability of line length, it appears to make a strong case that the origin of the MüllerLyer illusion is attributable to cognitive processes, rather than to structural ones.

\section{Requirements of a Detection-Theoretic Study of the Müller-Lyer Illusion}

With some refinements, Nevin's (1991) design can provide a detection-theoretic analysis of the Müller-Lyer illusion. One refinement is to arrange the stimuli so that the same standard line appears in both kinds of trials, illusory and nonillusory (see Table 3 ). Judgments about a comparison line that gives rise to an illusory effect can then be compared with judgments about a comparison line that does not give rise to an illusion. The comparison lines are not directly compared with each other, but rather with a common standard line. This is an important difference from the arrangement employed by Nevin. This refinement is based on the assumption that the sensory events of the standard line and of the comparison line are Gaussian and independent. DeLucia (1993) offers some support for the assumption of independence. She showed that the magnitude of the total illusion is not significantly different from the sum of the magnitudes of its components parts-that is, from the sum of the illusions from lines with arrowheads and from lines with fins.

A second refinement is to obtain judgments that yield a criterion-free index of discrimination, such as $d^{\prime}$, from a full receiver-operating characteristic (ROC). A full ROC allows the goodness-of-fit of the detection-theory model to be assessed - an assessment that is unavailable, for example, when the yes-no method is used to yield only a single point in the ROC square, because any curve can pass through one point. An efficient method for this purpose is the rating method, in which observers are asked to rate their confidence that one line is longer than another. 
By presenting several comparison lines in an experimental session, as is done in the method of constant stim$\mathrm{uli}$, the ratings of one comparison line with reference to a standard line can be compared with the ratings of another comparison line with reference to the same standard. By this means the discriminability of any pair of lines can be found, even though the lines were not directly compared with each other. The discriminability of the lines can then be presented in separate psychometric functions for various configurations of the Müller-Lyer illusion as well as for nonillusory figures, and the magnitude of the illusion can be assessed by the horizontal separation between the appropriate criterion-free functions.

\section{METHOD}

\section{Subjects}

Six volunteers took part, 2 men and 4 women. They all had normal or corrected-to-normal vision.

\section{Apparatus and Stimuli \\ All stimulus figures (white on black) were displayed on a 14-in. VGA monitor with $640 \times 480$ resolution. Three kinds of horizon- tal lines were used: lines with short vertical bars at each end, lines with arrowheads at each end, and lines with tail fins at each end (see Figure 1). The sloping terminations subtended an angle of $45^{\circ}$ with respect to the horizontal line. All terminating elements were $15 \mathrm{~mm}$ long. On every trial, a pair of lines was presented side-by- side for $1.0 \mathrm{sec}$. The left-hand line was always $70 \mathrm{~mm}$ long and was always terminated by two vertical bars; it is designated the standard line. The right-hand line was ierminated with vertical bars, arrow- heads, or fins; it is designated the comparison line. The comparison line could be $64,66,68,70,72,74$, or $76 \mathrm{~mm}$ long. The standard and comparison lines were always separated by $38 \mathrm{~mm}$. \\ During an experimental session, only two configurations of the pairs of lines occurred. In one configuration (nonillusory), the com- parison line, like the standard line, had vertical bars at each end; in the other configuration (illusory), the comparison line had either arrowheads or tail fins at each end, depending on the experiment. In an experimental session, the two configurations, illusory or non- illusory, were presented with equal probability. Each of the seven comparison lengths was presented in random order in each experi- mental session.}

\section{Procedure}

There were two experiments. In Experiment 1, the comparison line in the illusory configuration had arrowheads at each end; in Experiment 2, the comparison line in the illusory configuration had tail fins at each end. The observers were seated at a viewing dis-
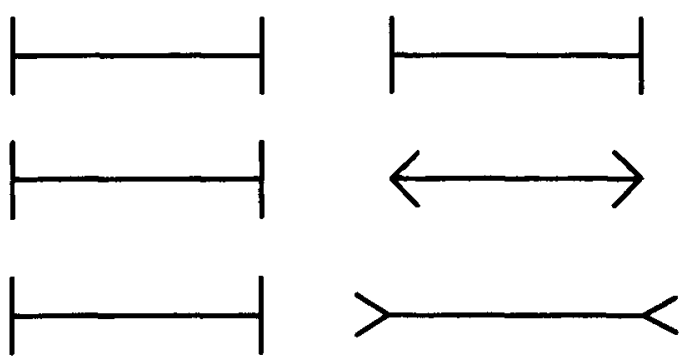

Figure 1. The top configuration was presented in both Experiments 1 and 2, the middle configuration in Experiment 1, and the bottom configuration in Experiment 2. tance of approximately $400 \mathrm{~mm}$ from the computer screen. On every trial, the standard line and a comparison line were presented together; after each observation, the observers rated their confidence, on a six-point scale, that the comparison line was longer or shorter than the standard. Their judgments were entered by pressing a numeric key on the computer's keyboard; 1 indicated that they were very confident that the comparison line was shorter than the standard, and 6 indicated that they were very confident that the comparison line was longer than the standard. Intermediate numbers represented intermediate degrees of confidence. No feedback was given, and the next trial began immediately after a response.

An experimental session comprised 112 trials, preceded by 12 warm-up trials that were discarded from the analysis. Each experiment entailed seven sessions, so there were $112 \times 7=784$ experimental trials per observer per experiment. All observers undertook both experiments (1,568 trials in all), but in counterbalanced order. During an experimental session, the seven comparison lengths for both illusory and nonillusory lines were each presented eight times in random order.

\section{Analysis}

ROCs were constructed from the ratings in the following way. For Experiment 1, an ROC for discriminating between the length of two lines, both with vertical bars at their ends, could be constructed from the ratings given to separate comparison lines with reference to the standard line. The technique is analogous to that used by Irwin and Whitehead (1991) and Irwin, Hautus, Dawson, Welch, and Bayly (1994). Second, an ROC could be constructed for discriminating between the length of two lines, one of which was terminated by arrowheads and the other by vertical bars, from the ratings given to the comparison line with arrowheads as opposed to those given to the comparison line with vertical bars. Third, an ROC could be constructed for discriminating between the lengths of two lines, both terminated by arrowheads, from the ratings given to each line in comparison with the standard line. These ROCs were available from the results of Experiment 1. An analogous set of ROCs for lines with and without tail fins was available from Experiment 2.

Table 3 illustrates a simplified version of the stimulus-response matrix for Experiment 1. The matrix is a simplification in that it supposes that only a binary response (longer or shorter) was allowed, whereas in fact a six-point rating was available. An important difference between our design and Nevin's (1991) is that, in our experiments, the same standard line appeared in both kinds of trials, nonillusory (S1) and illusory (S2). Because of this, the perceived length of any comparison line - for example, a line terminated with arrowheads-could be compared with the perceived length of another comparison line-for example, one terminated with finseven though they had not been judged with respect to each other.

Our experiments allowed six responses, not just two; for this reason, it was desirable to combine the ratings of the 6 observers in order to obtain average ROCs, because some of the ratings were used infrequently, especially for lines that were judged as being very different in length. This made it difficult to compute the path of an ROC for each observer separately. In order to construct ROCs for the 6 observers combined, a jackknifing procedure (see Dorfman \& Berbaum, 1986) was therefore implemented. This procedure avoids the pitfalls that can arise from the simple pooling of ratings when different criteria are adopted by different observers.

\section{RESULTS}

\section{Receiver-Operating Characteristics}

Figure 2 illustrates some of the ROCs obtained from each experiment. The three left-hand ROCs are from Experiment $l$ (where the comparison line in the illusory configuration was terminated with arrowheads), and the three 
Table 3

A Simplification of the Stimulus-Response Matrix for Experiment 1

\begin{tabular}{|c|c|c|c|c|}
\hline \multirow[b]{2}{*}{ Stimulus } & \multicolumn{2}{|c|}{ Stimulus } & \multicolumn{2}{|c|}{ Response } \\
\hline & Standard & Comparison & "Co longer" & "Co shorter" \\
\hline S1 & $\longmapsto$ & $\longmapsto$ & $p$ ("longer" $\mid \mathrm{S} 1$ ) & $p$ (“shorter"|S1) \\
\hline S2 & $\longmapsto$ & $\longleftrightarrow$ & $p$ ("longer"|S2) & $p$ ("shorter"|S2) \\
\hline
\end{tabular}

right-hand ROCs are from Experiment 2 (where the comparison line in the illusory configuration was terminated with tail fins). The data points are from the pooled ratings of all observers, and the theoretical ROCs are the best fits to the ratings of the normal-normal equal-variance model, which is the standard model of detection theory. The fits were obtained by maximum-likelihood estimation combined with the jackknifing technique. In these examples, each ROC shows the discriminability of a comparison line of $76 \mathrm{~mm}$ from a comparison line of $70 \mathrm{~mm}$. The ROCs were obtained from the ratings given to a comparison line of $76 \mathrm{~mm}$ and the ratings given to a comparison line of $70 \mathrm{~mm}$. Both these sets of ratings were made with respect to the same standard line of $70 \mathrm{~mm}$.

To clarify our analysis still further, consider Table 4, which shows some of the data from which the ROCs for Experiment 1 were constructed. The ratings of each row in the table were compared with the ratings given, in the same experimental session, to a comparison line of $70 \mathrm{~mm}$ that was terminated with vertical bars. The frequency of the six ratings to the $70-\mathrm{mm}$ comparison line with vertical bars turned out to be $2,27,238,302,83$, and 20 . Hence, the ROC depicted in the middle left-hand panel of Figure 2 was constructed by relating these ratings to those shown in Table 4 for the 76-mm line that was terminated with arrowheads. As another example, the ROC shown in the bottom left-hand panel was constructed from the ratings shown in Table $\mathbf{4}$ for the 76- $\mathrm{mm}$ line and from the ratings, also shown in Table 4, for the 70-mm line. Both these lines were terminated by arrowheads. Our procedure, however, did not provide an ROC for discriminating between two 70-mm lines when both were terminated in the same way, because only one set of ratings was available for this case.

The two top ROCs in Figure 2 illustrate independent estimates from Experiments 1 and 2 of the discriminability of two lines, one of $76 \mathrm{~mm}$ and the other of $70 \mathrm{~mm}$, that were terminated with vertical bars. The best-fitting values of $d$ 'for these two cases were 1.41 (Experiment 1) and 1.52 (Experiment 2). The other ROCs illustrate the effect of other terminations on the discriminability of two lines of these lengths, as illustrated in each panel (the configurations illustrated in the legend of each graph are not drawn to scale). The best-fitting $d^{\prime}$ for the left-hand middle panel has a negative value of -0.18 ; this signifies that, although the comparison line with arrowheads was longer than the comparison line with vertical bars (a line identical to the standard), it was judged to be slightly shorter-an example of the Müller-Lyer illusion. The right-hand middle panel illustrates the other side of the illusion, in which tail fins on the comparison line exaggerated the judged difference between the two lines $\left(d^{\prime}=2.57\right)$. The two bottom ROCs illustrate how arrowheads or tail fins on both lines affected their discriminability $\left(d^{\prime}=1.16\right.$ for the arrowheads and 0.89 for the tail fins).

\section{Psychometric Functions}

The six ROCs in Figure 2 illustrate the discriminability of a comparison line of $76 \mathrm{~mm}$ from a comparison line of $70 \mathrm{~mm}$. Altogether, seven different lengths of the comparison line were presented, however, so other ROCs of this type were constructed, and the best-fitting estimate of $d$ 'found for each. Figure 3 shows how, in Experiment 1 , these estimates depended on the difference in length between two lines when one of the lines was $70 \mathrm{~mm}$ long. (Only six points are shown when the two lines had identical terminations because, in this case, the method did not yield independent ratings for lines of equal length.) Figure 4 shows the comparable results for Experiment 2. In these psychometric functions, a negative $d^{\prime}$ means that the comparison line was perceived to be shorter than another line of $70 \mathrm{~mm}$. The lines fitted to each set of points are least-squares linear functions with two free parameters-intercept and slope.

The psychometric functions in Figures 3 and 4 have the form $d^{\prime}=m x+c$, where $x$ is the difference in length between the comparison line and a line of $70 \mathrm{~mm}, m$ is the slope of the psychometric function, and $c$ is its intercept. The constant error is therefore equal to $-c / m$, which is the value of $x$ when $d^{\prime}=0$. For an illusory configuration, the constant error specifies the magnitude and direction of the illusion. For the comparison lines with arrowheads (the triangles in Figure 3), the constant error was $7.8 \mathrm{~mm}$, and for the comparison lines with tail fins (inverted triangles in Figure 4), the constant error was $-9.1 \mathrm{~mm}$. The size of the illusion therefore amounted to $11 \%$ and $13 \%$ for the two configurations. The constant errors for the two estimates of the nonillusory configuration (circles in Figures 3 and 4) were calculated to be $0.1 \mathrm{~mm}$ and $-0.7 \mathrm{~mm}$.

The slope of a psychometric function can provide an estimate of the just noticeable difference for discriminating between the length of two lines. If the just noticeable difference is defined as the difference between two comparison lines that yields $\Delta d^{\prime}=1$ (equivalent to $76 \%$ correct in a two-alternative forced-choice experiment), the reciprocal of the slope of the fitted function is the just noticeable difference. The estimates from the two experiments of the slope of the psychometric functions when the comparison line, like the standard line, was terminated by vertical bars are 0.25 and $0.20 \mathrm{~mm}^{-1}$, giving two estimates of the just noticeable difference of $4.0 \mathrm{~mm}$ and $5.0 \mathrm{~mm}$ for a standard line of $70 \mathrm{~mm}$. The slope of the psychometric function when the comparison line was terminated with the arrowheads (Experiment 1 ) is 0.16 , which corresponds to a just noticeable difference of 

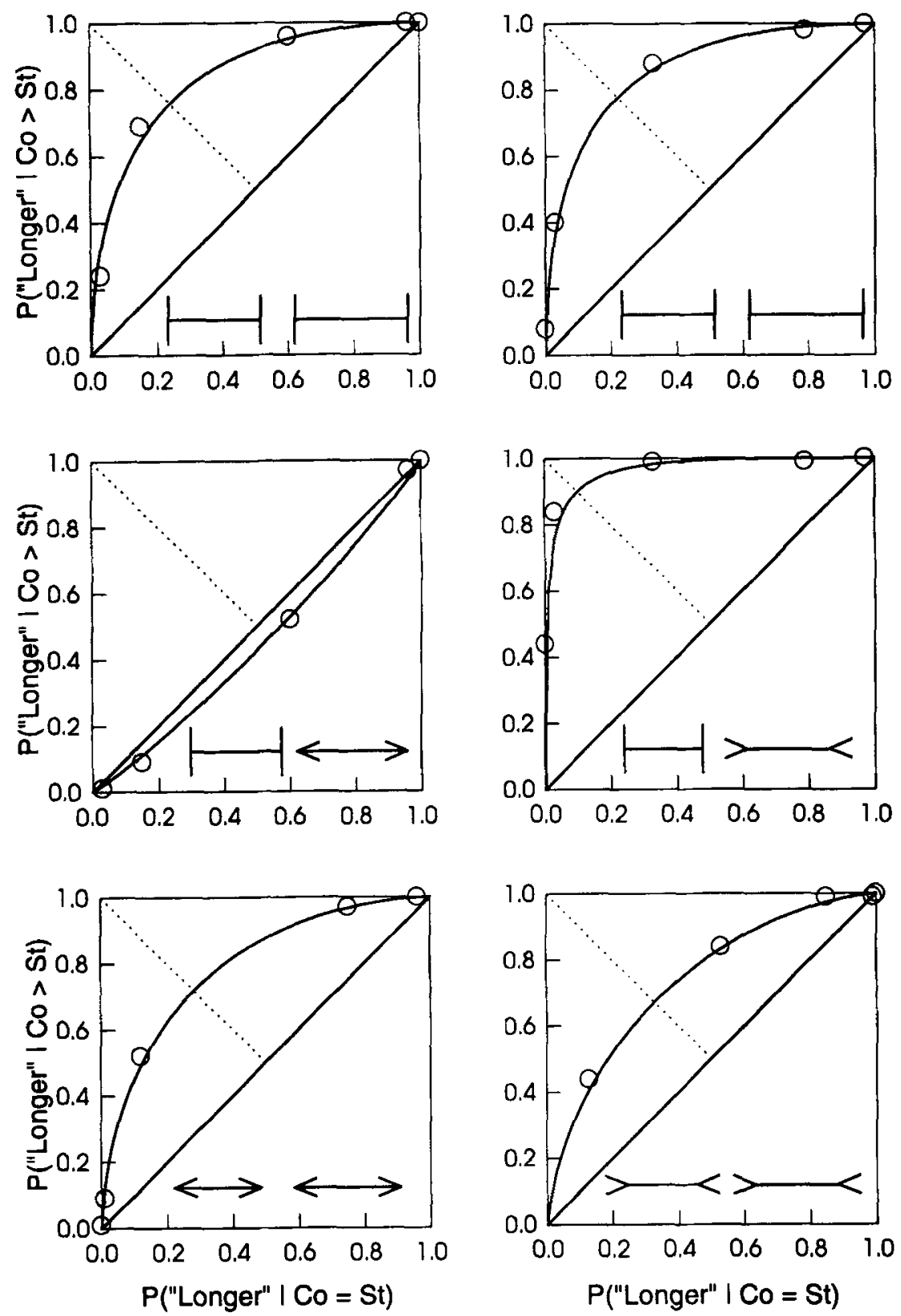

Figure 2. The curves show the best-fitting ROCs (normal-normal equal-variance model) to the obtained ratings for the lines illustrated in each panel. The lengths of the two lines were $70 \mathrm{~mm}$ and $76 \mathrm{~mm}$.

$6.3 \mathrm{~mm}$. When the comparison line was terminated with tail fins (Experiment 2), the slope is 0.17 , which corresponds to a just noticeable difference of $5.9 \mathrm{~mm}$ All these just noticeable differences are for lines presented for $1.0 \mathrm{sec}$, which did not allow prolonged inspection.

Figures 3 and 4 each show a third psychometric function for the discrimination between two lines that were both terminated with arrowheads (Figure 3 ) or both terminated with tail fins (Figure 4). Inspection of Figures 3 and 4 suggests that the slope of the function for discriminating between two lines that are terminated with verti- cal bars is steeper than are the slopes of the psychometric functions when one or both of the lines had sloping terminations. An analysis of variance (ANOVA) confirms this. The slopes of the psychometric functions of Experiment 1 in Figure 3 are not the same, for the interaction between the three types of termination and the six differences in length was significant $[F(10,50)=3.78, p<$ .001 ]; the same result was found for Experiment 2 (Figure 4) $[F(10,50)=4.35, p<.001]$. (In these analyses, the error term was estimated from the pseudovalues returned by the jackknife.) Hence the just noticeable differ- 
Table 4

Frequency With Which Each of Seven Comparison Lines, Terminated With Arrowheads, Were Rated Longer Than a Standard 70-Millimeter Line Terminzted by Vertical Bars

\begin{tabular}{crrrrrrr}
\hline & \multicolumn{7}{c}{ Rating } \\
\cline { 2 - 6 } Comparison $(\mathrm{mm})$ & \multicolumn{1}{c}{1} & \multicolumn{1}{c}{2} & 3 & 4 & 5 & 6 & Sum \\
\hline 64 & 152 & 354 & 143 & 16 & 3 & 4 & 672 \\
66 & 96 & 304 & 238 & 29 & 3 & 2 & 672 \\
68 & 51 & 224 & 352 & 39 & 4 & 2 & 672 \\
70 & 26 & 142 & 421 & 78 & 5 & 0 & 672 \\
72 & 7 & 102 & 432 & 121 & 10 & 0 & 672 \\
74 & 8 & 52 & 385 & 203 & 20 & 4 & 672 \\
76 & 2 & 20 & 299 & 292 & 49 & 10 & 672 \\
\hline
\end{tabular}

Note-The ratings are pooled from 6 observers.

ence in length of two lines that were terminated by vertical bars was smaller than the just noticeable difference in length of lines that were terminated in other ways.

Another question of interest is whether the slopes of the four psychometric functions for lines that are terminated with sloping elements differ among themselves. An ANOVA showed that the interaction between four types of sloping element and the six differences in length was not significant $[F(15,75)=0.41$, n.s. $]$.

The shape of a psychometric function that is linear in $d^{\prime}$ assumes a different form when discriminability is measured in units of proportion correct $[p(c)]$. The relation between $d^{\prime}$ and $p(c)$ for an unbiased observer is $p(c)=\Phi\left(d^{\prime} / \sqrt{2}\right)$, where $\Phi()$ is the normal distribution function. Hence, linear psychometric functions like those in Figures 3 and 4 can be represented by the normal distribution function when discriminability is specified in units of $p(c)$. Indeed, this is the expected theoretical shape of such a psychometric function when the stimuli being discriminated are presented separately, as they are here (see Irwin, 1989; Laming, 1986).

\section{DISCUSSION}

The results show that the Müller-Lyer configuration causes a change in the discriminability of the length of its two lines, a change that is manifested as a constant error. Because we measured discriminability by ROC analysis, this result cannot be attributed to changes in response bias, for the ROC contains all possible biases for a given discriminability. In this respect, our conclusion is the opposite of that of Nevin (1991), who attributed the illusion to changes in response bias and not to discriminability. Although our conclusion differs from Nevin's, we do not think that Nevin's results are necessarily in conflict with our own. Although Nevin's psychometric functions control for response bias, their slopes measure the discriminability of two lines with the same terminations. Moreover, the displacement between two such psychometric functions is akin to a classical index of the illusion (see, e.g., Restle \& Decker, 1977), an index which does not allow the contribution of response bias and discriminability to be separated.
An examination of the middle left-hand ROC in Figure 2 shows that a $76-\mathrm{mm}$ line that is terminated by arrowheads is barely discriminable in length from a $70-\mathrm{mm}$ line that is terminated by vertical bars $\left(d^{\prime}=0.18\right)$. By contrast, the same two lines terminated by vertical bars are more readily discriminable - as is shown, for example, by the top left-hand ROC in Figure 2, for which $d^{\prime}=$ 1.41. Our results, therefore, do not support strategy theories, insofar as those theories attribute the illusion, as Nevin (1991) has stated, to biasing factors stemming from criterion placement.

Our results also show that the way in which the lines are terminated affects the slope of their psychometric function and, therefore, the size of the just noticeable difference in length, irrespective of any illusory effect that the terminations may produce. We found that the slope of a psychometric function for two lines that were terminated by vertical bars was significantly greater than the slope of psychometric functions for two lines that were terminated by other configurations (see Figures 3 and 4). Two of these psychometric functions were for illusory configurations (one line terminated with vertical bars and the other line terminated with either arrowheads or tail fins) and two were for nonillusory configurations (both lines terminated with arrowheads or both with tail fins). The difference may stem from the fact that vertical bars provide more information on the length of the lines, or a clearer definition of their ends, than do sloping terminations. Judd (1899) attributed the Müller-Lyer illusion to

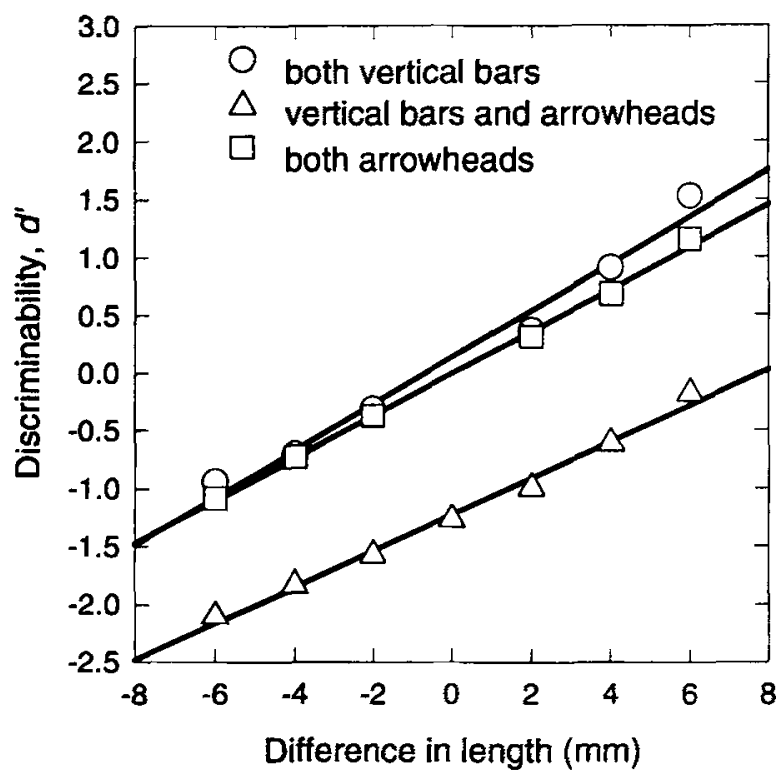

Figure 3. Psychometric functions from Experiment 1 showing the discriminability of two lines as a function of their difference in length. One line was always $70 \mathrm{~mm}$ long. The different symbols indicate the terminations of the lines: both lines terminated by vertical bars (circles), both terminated by arrowheads (squares), or one line terminated by vertical bars and the other by arrowheads (triangles). 


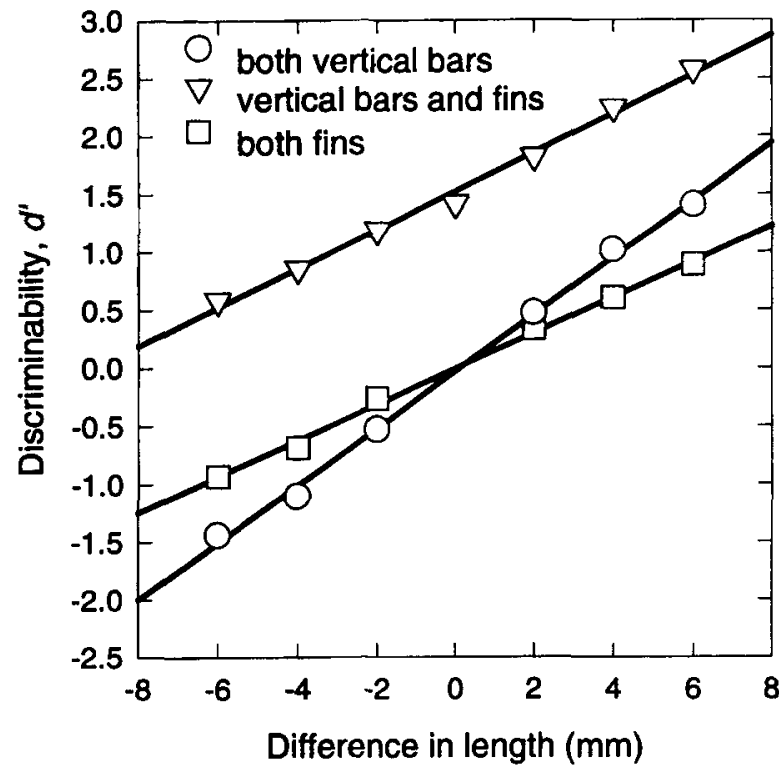

Figure 4. Psychometric functions from Experiment 2 showing the discriminability of two lines as a function of their difference in length. One line was always $70 \mathrm{~mm}$ long. The different symbols indicate the terminations of the lines: both lines terminated by vertical bars (circles), both terminated by fins (squares), or one line terminated by vertical bars and the other by fins (inverted triangles).

a similar process, but oui evidence on this point is confined to studying how the slope of the psychometric function for discriminating the length of two lines with identical terminations is affected by those terminations. Again, this conclusion differs from Nevin's (1991), for he reported no effect of the angular orientation of the elements of a terminating arrowhead, including an angle of $90^{\circ}$ that formed a vertical bar, on the slope of the psychometric function.

A related investigation by Morgan, Hole, and Glennerster (1990) found that the just noticeable difference in the length of a line was unaffected by its being embedded in a longer line with tail fins that could give rise to the Müller-Lyer illusion. From this they concluded that the illusion did not alter an observer's sensitivity to changes in line length. We found, by contrast, that the just noticeable difference in the length of a line was affected by the nature of its terminations. The task that Morgan et al. set for their observers was different from ours. Their observers had to judge the length of part of the shaft (a segment marked by vertical lines) embedded in a longer line with tail fins, whereas our observers judged the whole shaft, which was sometimes in an illusory configuration and sometimes not. Morgan et al. used vertical markers to delineate the line segment to be judged, and it may be that this procedural variation gives rise to the difference between their results and ours. Moreover, Morgan et al. did not study the effect of different terminations on the just noticeable difference for line length, and so, in this respect, they did not consider the same question that we did.

\section{CONCLUSION}

We have shown how a refinement of Nevin's (1991) design can provide a detection-theoretic analysis of the Müller-Lyer illusion. Our results show that the MüllerLyer figure causes a change in the discriminability of the length of its lines; therefore, the illusion cannot be attributable solely to response biases or to any processes that are reflected in response biases. However, this analysis cannot offer a precise statement about the origin of the illusion. It does not pinpoint whether, to use Coren and Girgus's (1978) classification, the structural origin is optical, retinal, or cortical. In this sense, our work, following Nevin's lead, illustrates how a detection-theoretic analysis can be made of the Müller-Lyer and other illusions but does not offer a definitive explanation of them.

\section{REFERENCES}

Brosvic, G. M., Civale, N. A., Long, P., Kieley, K. K., Memblatt, N., Gordon, R., Parris, L., \& Giambelluca, C. (1994). Signal-detection analysis of the Müller-Lyer and the horizontalvertical illusions. Perceptual \& Motor Skills, 79, 1299-1304.

COREN, S., \& GiRGus, J. S. (1978). Seeing is deceiving: The psychology of visual illusions. Hillsdale, NJ: Erlbaum.

DAY, R. H., \& KNUTH, H. (1981). The contributions of F. C. MüllerLyer. Perception, 10, 126-146.

DeLucia, P. R. (1993). A quantitative analysis of illusion magnitude predicted by several averaging theories of the Müller-Lyer illusion. Perception \& Psychophysics, 53, 498-504.

Dorfman, D. D., \& Berbaum, K. S. (1986). RSCORE-J: Pooled ratingmethod data: A computer program for analyzing pooled ROC curves. Behavior Research Methods, Instruments, \& Computers, 18, 452-456.

GINSBURG, A. P. (1986). Spatial filtering and visual form perception. In K. R. Boff, L. Kaufman, \& J. P. Thomas (Eds.), Handbook of perception and human performance: Vol. 2. Cognitive processes and performance (pp. 34.1-34.41). New York: Wiley.

IRWIN, R. J. (1989). Psychometric functions for the discrimination of differences in intensity of Gaussian noise. Quarterly Journal of Experimental Psychology, 41A, 655-674.

IrWin, R. J., Hautus, M. J., Dawson, N. J., Welch, D., \& Bayly, M. F. (1994). Discriminability of electrocutaneous stimuli after topical anesthesia: Detection-theory measurement of sensitivity to painful stimuli. Perception \& Psychophysics, 55, 125-132.

IRWIN, R. J., \& WHITEHEAD, P. R. (1991). Towards an objective psychophysics of pain. Psychological Science, 2, 230-235.

JuDD, C. H. (1899). A study of geometrical illusions. Psychological Review, 6, 241-261.

LAMING, D. (1986). Sensory analysis. London: Academic Press.

Lown, B. A. (1988). Quantification of the Müller-Lyer illusion using signal detection theory. Perceptual \& Motor Skills, 67, 101-102.

Morgan, M. J., Hole, G. J., \& Glennerster, A. (1990). Biases and sensitivities in geometrical illusions. Vision Research, 30, 1793-1810.

NEVIN, J. A. (1991). Signal-detection analysis of illusions and heuristics. In M. L. Commons, J. A. Nevin, \& M. C. Davison (Eds.), Signal detection: Mechanisms, models, and applications (pp. 257-274). Hillsdale, NJ: Erlbaum.

Pressey, A. W., \& Pressey, C. A. (1992). Attentive fields are related to focal and contextual features: A study of Müller-Lyer distortions. Perception \& Psychophysics, 51, 423-436.

Restle, F., \& DeCKER, J. (1977). Size of the Mueller-Lyer illusion as a function of its dimensions: Theory and data. Perception \& Psychophysics, 21, 489-503.

(Manuscript received February 5, 1996; revision accepted for publication February 23, 1997.) 